Editorial

\title{
The mechanics of the brain
}

\author{
Andreas Draguhn ${ }^{1, *}$ \\ ${ }^{1}$ Institute for Physiology and Pathophysiology, Medical Faculty, Heidelberg University, 69120 Heidelberg, Cermany \\ *Correspondence: andreas.draguhn@physiologie.uni-heidelberg.de (Andreas Draguhn)
}

DOI:10.31083/j.jin2101022

This is an open access article under the CC BY 4.0 license (https://creativecommons.org/licenses/by/4.0/).

Submitted: 5 January 2022 Revised: 14 January 2022 Accepted: 14 January 2022 Published: 28 January 2022

The brain is an organ. This apparently trivial notion is sometimes neglected in the language of basic neurosciences, which tend to resort to metaphors from electrical engineering, computer sciences and information theory. However, the brain is not a computer, but is embedded within a whole body, giving rise to multiple bi-directional interactions. Over recent years, network-level, behavioral and cognitive neuroscientists have developed increasing interest in these brainbody interactions, with special emphasis on rhythmic activity patterns like cardiovascular activity [1], respiration [2, 3] and gut motility [4].

The recent paper by Wang and Hamill [5] adds an interesting molecular perspective to these developments: they describe the widespread occurrence of a mechanosensitive cation channel, PIEZO2, in cortical neurons of the mouse brain. PIEZO channels have been discovered as late as in 2010 by the laboratory of Ardem Patapoutian at Scripps Research [6]. This finding, acknowledged by the Nobel Prize for Physiology or Medicine in 2021, revealed an entirely new class of ion channels with unique molecular properties optimized for transducing membrane tension into electrical (depolarization) and biochemical ( $\mathrm{Ca}^{2+}$-dependent processes) signals (Fig. 1, Ref. [6]). It soon became clear that PIEZO channels are crucial for multiple mechanical transduction processes including regulation of local blood flow, systemic blood pressure, depth of breathing and also cell differentiation and growth in various tissues. One of the two mammalian channel subtypes, PIEZO2, turned out to be the molecular correlate of touch perception, which had been elusive for decades [7].

Most studies to date have focused on the presence and activity of PIEZO channels in peripheral tissues. There have been, however, several reports on their expression in the central nervous system, some of which are related to mechanical signals. For example, expression of PIEZO2 in the rat brain is upregulated after mechanical trauma [8]. Moreover, Hamill and coworkers have shown that pressure-activated conductance changes in cortical pyramidal neurons are capable of triggering action potentials. The underlying ion channels share several key properties with PIEZO channels [9].

Amid these discoveries, the expression pattern of PIEZO2 in the mouse brain remained largely unclear, with important questions outstanding on cell specificity. Do we find the channel in principal cells or in local circuit neurons? Does it occur in glial cells? Is it only present in defined subsets of a given type? To address these and related questions, Wang and Hamill [5] designed a new antibody directed against a highly conserved extracellular portion of the human PIEZO2 channel. The respective fragment had roughly $85 \%$ homology to the mouse homolog, and only $\sim 35 \%$ to PIEZO1. Indeed, control experiments proved specificity for PIEZO2 in mouse tissue, demonstrating that they had developed the right tool. Using this, they studied the expression of the channel in different networks, focusing on hippocampus, neocortex, cerebellum and olfactory bulb. Dorsal root ganglia, a peripheral tissue, were also included.

In essence, Wang and Hamill report the presence of PIEZO2 in principal neurons within all studied areas. Widespread expression was seen in neocortical pyramidal neurons of all layers (except the largely cell-free layer I), pyramidal neurons of selected hippocampal areas (mostly CA3 and CA2), cerebellar Purkinje cells, and mitral cells of the olfactory bulb. In dorsal root ganglia, they found an almost ubiquitous expression of PIEZO2 in ganglion cells, which form the major class of neurons connecting peripheral sensory receptors with central networks in the spinal cord. Several findings of the study are remarkable: (i) PIEZO2 expression seems to be most pronounced in typical projection neurons and far less in local circuit neurons; (ii) there is little evidence for expression in glia or peripheral satellite cells; (iii) staining of single cells appeared to follow an all-or-none pattern (which the authors interpret with some caution, as the enzyme-mediated DAB staining protocol yields no linear relationship between staining intensity and antigen expression level). It will be interesting to see in future studies whether and how expression levels of PIEZO channels are regulated in central neurons. In any case, PIEZO2 seems to be present in a large but selective group of projection neurons throughout the mouse brain.

What are the functional implications of the present findings? At first glance, one might wonder why the brain would need mechanosensitive ion channels. After all, it is particularly well protected against all mechanical irritations by the cranial cavity and the surrounding fluid. There are, however, small but significant pressure fluctuations in the brain, driven by the oscillating amplitude of blood pressure, and 

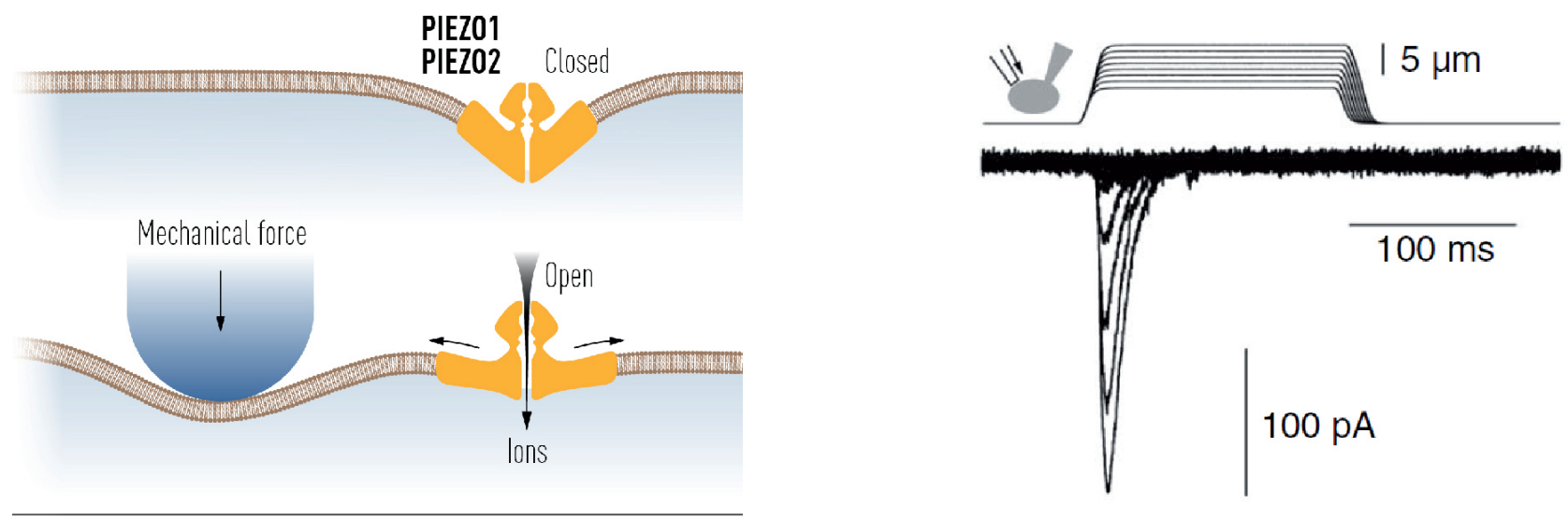

Fig. 1. Activation of PIEZO2. Left: Schematic representation of mechanical channel gating. Taken, with permission, from Advanced information, NobelPrize.org., Nobel Prize Outreach AB 2022, Mon. 3 Jan 2022. https://www.nobelprize.org/prizes/medicine/2021/advanced-information/). Right: Membrane currents mediated by PIEZO2 upon mechanical stimulation of the cell membrane. Taken, with permission, from Ref [6].

by respiration [10]. This point underscores the brain's embedment within the body-pressure pulsations may constitute an important example of body-to-brain signaling. Wang and Hamill argue that PIEZO2 is sufficiently sensitive to capture these pressure changes and translate them into rhythmic modulation of neuronal activity throughout the brain $[5,9]$. The unexpected expression in mitral cells might shed new light on the synchronization of brain activity by nasal respiration which is not necessarily coupled to specific olfactory inputs $[2,3]$.

But what benefit could be gained by the brain accounting for blood pressure pulsations or the mechanics of respiration? Wang and Hamill discuss two non-exclusive possibilities. First, frequency and intensity of both pulse and respiration vary with emotional or behavioral states, and so sensing these parameters might provide important information. This mechanism is reminiscent of the James-Lange theory from the late 19th century, proposing that emotions are essentially perceptions of somatic states $[11,12]$. While this theory has underestimated the role of environmental cues and brain-endogenous mechanisms, somatic feedback signals do indeed contribute to emotions $[4,13]$. Secondly, Wang and Hamill argue that the brain may simply make use of heartbeat or respiration as synchronizing signals, irrespective of their original biological function. Synchronous, rhythmic activity is a hallmark of neuronal networks, and it may be essential to allow information processing by coordinated multineuronal activity patterns $[14,15]$. Thus, small intracranial pressure fluctuations may be used to generate brain-wide coherent activity. Indeed, recent evidence shows that heartbeat and respiration do generate such widespread network synchrony which, in turn, entrains neuronal discharge behavior [1-3]. Wang and Hamill call this hypothetical evolutionary mechanism 'Intrinsic Resonance Discharge'. With respect to respiration, they provide an elegant sketch (Figure 7) on how such resonance mechanisms could interact with 'Res- piratory Corollary Discharge' from the rhythm-generating brainstem networks and with sensory feedback from nasal respiration ('Olfactory Re-afferent Discharge') to generate the widespread respiratory oscillations observed in the brains of rodents and humans [2, 3].

Thus, the brain-wide neuronal expression of PIEZO2 may turn out to be an important mediator between somatic physiology and cognitive, emotional or behavioral functions. The work of Wang and Hamill reminds us how intricately brain and body interact. This concept may well have been recognized and used in psychosomatic interventions since the beginning of medicinal practice. Elucidating the molecular and cellular foundations of these interactions may be a crucial step for modern medicine, just as it is certainly important to understand the function of the embedded and embodied brain.

\section{Ethics approval and consent to participate}

Not applicable.

\section{Acknowledgment}

Not applicable.

\section{Funding}

This research received no external funding.

\section{Conflict of interest}

The authors declare no conflict of interest.

\section{References}

[1] Schandry R, Montoya P. Event-related brain potentials and the processing of cardiac activity. Biological Psychology. 1996; 42: 7585.

[2] Heck DH, McAfee SS, Liu Y, Babajani-Feremi A, Rezaie R, Freeman WJ, Wheless JW, Papanicolaou AC, Ruszinkó M, Sokolov Y, Kozma R. Breathing as a Fundamental Rhythm of Brain Function. Frontiers in Neural Circuits. 2017; 10: 115.

[3] Tort ABL, Brankačk J, Draguhn A. Respiration-Entrained Brain 
Rhythms are Global but often Overlooked. Trends in Neurosciences. 2018; 41: 186-197.

[4] Azzalini D, Rebollo I, Tallon-Baudry C. Visceral Signals Shape Brain Dynamics and Cognition. Trends in Cognitive Sciences. 2019; 23: 488-509.

[5] Wang J, Hamill OP. Piezo2-peripheral baroreceptor channel expressed in select neurons of the mouse brain: a putative mechanism for synchronizing neural networks by transducing intracranial pressure pulses. Journal of Integrative Neuroscience. 2021; 20: 825-837.

[6] Coste B, Mathur J, Schmidt M, Earley TJ, Ranade S, Petrus MJ, et al. Piezo1 and Piezo2 are essential components of distinct mechanically activated cation channels. Science. 2010; 330: 55-60.

[7] Kefauver JM, Ward AB, Patapoutian A. Discoveries in structure and physiology of mechanically activated ion channels. Nature. 2020; 587: 567-576.

[8] Heyburn L, Abutarboush R, Goodrich S, Urioste R, Batuure A, Statz J, et al. Repeated Low-Level Blast Overpressure Leads to Endovascular Disruption and Alterations in TDP-43 and Piezo2 in a Rat Model of Blast TBI. Frontiers in Neurology. 2019; 10: 766.
[9] Nikolaev YA, Dosen PJ, Laver DR, van Helden DF, Hamill OP. Single mechanically-gated cation channel currents can trigger action potentials in neocortical and hippocampal pyramidal neurons. Brain Research. 2015; 1608: 1-13.

[10] Wagshul ME, Eide PK, Madsen JR. The pulsating brain: a review of experimental and clinical studies of intracranial pulsatility. Fluids and Barriers of the CNS. 2011; 8: 5 .

[11] James W. II (1884) What is an emotion? Mind. 1884; ox-IX: 188205.

[12] Lange CG. The mechanism of the emotions. The Emotions (pp. 33-92). In Dunlap D, (Ed.) Williams \& Wilkins: Baltimore, MD, USA. 1885.

[13] Klein AS, Dolensek N, Weiand C, Gogolla N. Fear balance is maintained by bodily feedback to the insular cortex in mice. Science. 2021; 374: 1010-1015.

[14] Buzsáki G, Draguhn A. Neuronal oscillations in cortical networks. Science. 2004; 304: 1926-1929.

[15] Panzeri S, Macke JH, Gross J, Kayser C. Neural population coding: combining insights from microscopic and mass signals. Trends in Cognitive Sciences. 2015; 19: 162-172. 